\title{
Microstructure and nanomechanical properties of the wing membrane of dragonfly
}

\author{
F. Song ${ }^{\text {a, } *}$, K.W. Xiao ${ }^{\text {a }}$, K. Bai ${ }^{\text {b }}$, Y.L. Bai ${ }^{\text {a }}$ \\ a State Key Laboratory of Nonlinear Mechanics (LNM), Institute of Mechanics, Chinese Academy of Sciences, \\ Beijing 100080, People's Republic of China \\ ${ }^{\mathrm{b}}$ Department of Chemical Biology, School of Pharmaceutical Sciences, Peking University Health Science Center, \\ Beijing 100083, People's Republic of China
}

Received 17 July 2006; received in revised form 5 December 2006; accepted 25 January 2007

\begin{abstract}
Detailed investigations on the microstructure and the mechanical properties of the wing membrane of the dragonfly were carried out. It was found that in the direction of the thickness the membrane was divided into three layers rather than as traditionally considered as a single entity, and on the surfaces the membrane displayed a random distribution rough microstructure that was composed of numerous nanometer scale columns coated by the cuticle wax secreted. The characteristics of the surfaces were accurately measured and a statistical radial distribution function of the columns was presented to describe the structural properties of the surfaces. Based on the surface microstructure, the mechanical properties of the membranes taken separately from the wings of living and dead dragonflies were investigated by the nanoindentation technique. The Young's moduli obtained here are approximately two times greater than the previous result, and the reasons that yield the difference are discussed.
\end{abstract}

(c) 2007 Elsevier B.V. All rights reserved.

Keywords: Microstructure; Surface structure; Mechanical properties; Surface analysis; Membrane

\section{Introduction}

The wing membrane of insects is a natural biological membrane made mainly of structural proteins. The material and structural properties of the membranes, together with the venations, of insect wings are associated with the flight of the insects themselves [1-3]. Recently, Combes and Daniel [1,2] indicated that most membranes on insect wings undergo significant bending and twisting during flight, which may alter the direction and magnitude of aerodynamic force production, and the deformations of the wing membranes increase thrust production in some species by creating a force asymmetry between half-strokes, and can enhance lift production by allowing wings to twist and generate upward force throughout the stroke cycle. Therefore, the material and mechanical properties of the wing membrane, together with that of the wing vein, determine how the wing will change shape in response to these forces [1,2]. In addition, the membrane is not simply a barrier to the passage of air through the

\footnotetext{
* Corresponding author. Fax: +86 1062579511.

E-mail address: songf@lnm.imech.ac.cn (F. Song).
}

wing but, in some areas at least, has a structural role as a stressed skin, stiffening the framework of veins. And there may be local variation in the mechanical properties and, hence, in the structure of the membrane within the wing, with profound implications for its functioning in flight $[3,4]$. However, the basic material and mechanical properties on the membrane of the insect wings, as well as its microstructure, have not been understood very well [1-5]. For example, the average Young's module of the wing membrane of dragonflies was measured by the traditional tensile test method to be about $1 \mathrm{GPa}$ [8], and determined by the nanoindentation technique to be about $1.5 \mathrm{GPa}$ [9]. Therefore, the accurate measurements on the mechanical properties of the membrane of the dragonfly wings have remained unclear so far, although there have recently been a lot of investigations on the flight mechanism of the dragonfly and its applications to the microair vehicles [6,7].

Traditionally, the measurements of the mechanical properties of the wing membrane of insects mainly used the methods of tensile testing by some mechanical test machines $[4,5,8]$. However, the tensile testing is very inconvenient to some small scale samples of insect wings, such as measuring the mechanical properties of a cell (a compartment of membrane between wing veins) 
of dragonfly wings, the area of which is generally about $1 \mathrm{~mm}^{2}$. Moreover, the wing membranes of many insects are corrugated or pleated, at least in part. For example, those of Odonata are pleated almost throughout [8]. Therefore, it is necessary to find a new testing method that can more conveniently and accurately measure the mechanical properties of the small scale samples of insects such as the cells on the dragonfly wings. The nanoindentation technique is considered as an excellent tool for the study of the mechanical behavior of thin membranes, in particular, when simple tensile tests are very small and difficult to perform [9-12]. The development of the nanoindentation technique has allowed highly localized hardness and modulus measurements to be performed on very small material volumes. In principle, if a very sharp tip is used, the contact area between the sample and the tip, and thus the volume of material that is tested, can be made arbitrarily small $[10,13]$.

In the present study, our investigations focus on the microstructure and material properties of the wing membrane of the dragonfly, in particular, the differences of mechanical properties between the wing membranes of living and dead dragonflies. By means of scanning electron microscope (SEM), we measured the relevant geometrical and morphological characteristics of the microstructure of the membrane. Then, a statistical radial distribution function of the microstructure was presented to describe the geometrical characteristics of the surfaces of the membrane, and the hydrophobicity of the surfaces of the membrane was briefly investigated. Based on the microstructural characteristics, the Young's modulus and the hardness of the membrane were accurately measured by using the nanoindentation technique. Finally, we briefly gave some discussions on the microstructure and material characteristics of the membrane.

\section{Experimental}

\subsection{Materials and sample preparation}

Test samples of the wing membranes were taken from the dragonflies (Libellula basilinea McLachlan) that were caught in the suburb of Beijing. Each of the tested samples consisted only of one cell that was taken separately from $R, R_{2}, 1 s t R_{3}$, $2 \mathrm{ndR}_{3}, \mathrm{R}_{4}, \mathrm{R}_{5}, \mathrm{M}_{1}, 1 \mathrm{stM}_{2}, 2 \mathrm{ndM}_{2}, \mathrm{M}_{3}, \mathrm{M}, \mathrm{M}_{4}, \mathrm{Cu}_{1 \mathrm{a}}, \mathrm{Cu}_{1 \mathrm{~b}}$ and $1 \mathrm{~A}$ in the forewings of dragonflies designated by the ComstockNeedham system. We tested 15 different types of cells taking from 20 forewings of 10 dragonflies. Each type of the cells tested included 20 testing samples in which the living and the dead were separately 10 samples. The dead samples of the wing membranes were cut out from the wings of the dragonflies that had died before 3 days and were preserved in a container with room temperature and humidity. Based on the fact that the material properties of the wing membrane changed fairly rapidly after being removed from the insect [4], all living specimens of the membranes were directly removed from the wings of living dragonflies, rapidly installed and examined on the relevant machines within $5 \mathrm{~min}$. So that, the tested properties of the membranes were deemed approximately the same as that of the parts of a living dragonfly.

\subsection{Microstructure measurements}

We employed a SEM (Sirion 400NC, FEI, USA) to observe and measure the microstructure of the wing membrane of the dragonfly. Since the thickness of the wing membranes was very thin, to measure the microstructure along the thickness of the membrane, we dipped the tested samples in liquid nitrogen a few minutes so that the membranes became very brittle, took out the samples from the liquid nitrogen and broke them to obtain fresh and planar fracture cross-sectional surfaces of the membranes in the direction of the thickness. After that, all the treated samples were coated with gold about $8 \mathrm{~nm}$ thick, and then, the samples were observed by using SEM. Finally, all tested data of SEM images were analyzed and recorded by employing Image Analysis System (IAS, Image-Pro Plus 4.5, Media Cybernetics).

\subsection{Mechanical testing}

Nanoindentation tests of the wing membranes were carried out by using a MTS NanoIndenter XP (MTS Systems Corp., Oak Ridge, TN, USA) with a Berkovich diamond tip, and the hardness and the Young's modulus of the membranes were obtained by using the continuous stiffness measurement (CSM) option. We chose an aluminium plate as testing substrate and used an instant adhesive (502 adhesive) to glue the tested sample on it. Note that the adhesive between the tested membrane and the substrate should be paved as thin and uniform as possible for determining accurately the Young's modulus and the hardness of the membrane [14]. In our experiments, we used the Berkovich diamond tip of the nanoindenter to penetrate the two layers of the membrane and the adhesive from the surface of the membrane to the surface of the aluminium substrate, such that, we can obtained the total thickness of the tested membrane and adhesive. Therefore, the thickness of the adhesive layer was estimated to be about the difference between the total thickness that the tip penetrated and the thickness of the membrane. The average thickness of the adhesive layers in our experiments was roughly equal to $0.42 \pm 0.15 \mu \mathrm{m}$, which was satisfied the requirement of the nanoindentation.

According to the theory of nanoindentation test $[10,13,14]$, the reduced modulus of the tested materials is expressed as

$E_{\mathrm{r}}=\frac{\sqrt{\pi}}{2 \beta} \frac{S}{\sqrt{A}}$

where $S$ is the contact stiffness that is defined by $S=(\mathrm{d} P / \mathrm{d} h)_{\text {unload }}$, and $\beta$ is a coefficient related to the geometry of the tip, $\beta=1.034$ for the Berkovich tip. However, it is usually difficult to obtain the contact stiffness according to the definition. Employing the CSM method, we have

$S=\frac{1}{\left(1 /\left(\left(P_{0} / Z_{0}\right) \cos \Phi-\left(K_{\mathrm{S}}-m \omega^{2}\right)\right)\right)-\left(1 / K_{\mathrm{f}}\right)}$

where $P_{0}$ is the amplitude of the harmonic force, $Z_{0}$ the amplitude of the tip's displacement, and $\Phi$ is the phase angle between the displacement and the force. In this equation, $K_{\mathrm{S}}$ and $K_{\mathrm{f}}$ is the corresponding values of the nanoindenter, $m$ the mass of the 
indentation system, and $\omega$ is the angular frequency of the harmonic load, are constants of the machine, and $P_{0}, Z_{0}$ and $\Phi$ are automatically measured and recorded by the nanoindenter. So, the contact stiffness $S$ is determined by Eq. (2).

Usually, the indented area is difficult to be measured by microscope. Thus, the load and displacement during indentation process are recorded and these data are analyzed to obtain the contact area. The contact area, $A$, is related to the contact depth $h_{\mathrm{c}}$, which can be written as

$A=\sum_{n=0}^{8} C_{n} h_{\mathrm{c}}^{1 / 2^{n-1}}$

where $C_{n}$ are constants that depend on the indenter geometry and are given by calibrating initially a standard sample with known material properties $[15,16]$. In the present study, $C_{0}=21.4, C_{1}=2100$, and take the rests to be zero. In Eq. (3), $h_{\mathrm{c}}$ is determined by

$h_{\mathrm{c}}=h_{\max }-\varepsilon \frac{P_{\max }}{S}$

For the Berkovich tip, $\varepsilon=0.75$ is a constant depending on the indenter geometry. The relationship between indentation load, $P$, and penetration depth, $h$, is as shown in Fig. 1, where the maximum load, $P_{\max }$, and maximum penetration depth, $h_{\max }$, are recorded automatically by the indenter. From Eqs. (1)-(4), we can obtain the reduced modulus $E_{\mathrm{r}}$.

Further, the effects of a non-rigid indenter on the load displacement behavior can be taken into account by defining an effective modulus, $E_{\mathrm{r}}$, as follows

$\frac{1}{E_{\mathrm{r}}}=\frac{1-v^{2}}{E}+\frac{1-v_{\mathrm{i}}^{2}}{E_{\mathrm{i}}}$

where $E$ is the Young's modulus and $v$ is the Poisson's ratio of the specimen; $E_{\mathrm{i}}$ and $\nu_{\mathrm{i}}$ are the corresponding values of the

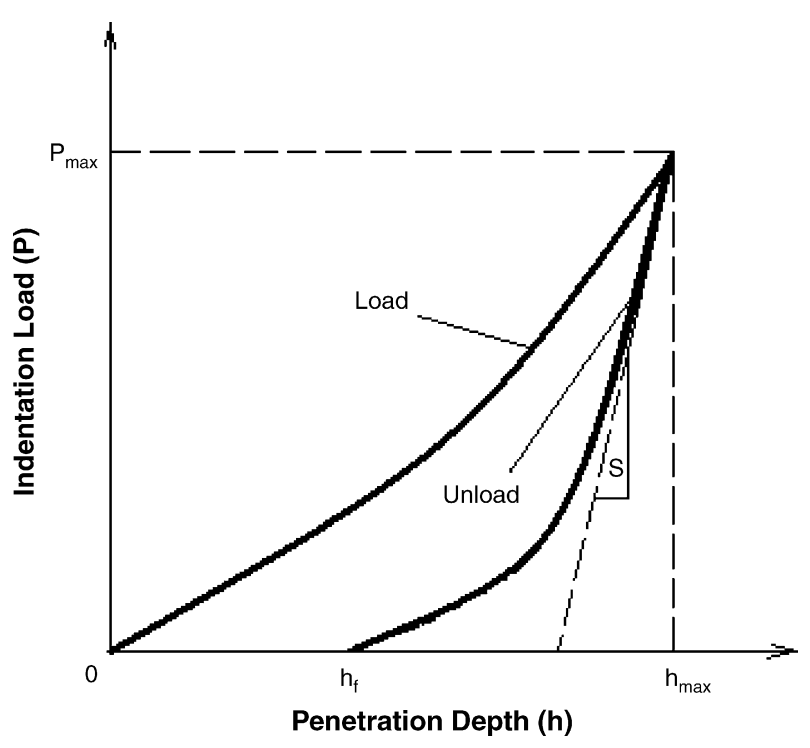

Fig. 1. Plot of indention load, $P$, and penetration depth, $h$, showing the process of the loading and unloading of the nanoindenter. indenter. For the diamond indenter used in our experiments, $E_{\mathrm{i}}=1141 \mathrm{GPa}$ and $v_{\mathrm{i}}=0.07$. Also, in the present calculation, Poisson's ratio of the specimen is taken as 0.4 for macromolecular materials [14-16]. The hardness of the material is defined by

$H=\frac{P_{\max }}{A}$

According to Eqs. (1)-(6), Young's modulus and the hardness of the tested materials can be obtained. Note that in the testing process of the wing membrane by virtue of CSM method the indenter was firstly loaded to a peak load and held at the peak load for $10 \mathrm{~s}$, then unloaded $90 \%$ from the peak load and held the load for $50 \mathrm{~s}$ for thermal drift correction, finally, unloaded completely. The strain rate and the allowable thermal drift rate were taken in the testing process to be $0.05 \mathrm{~s}^{-1}$ and $0.05 \mathrm{~nm} / \mathrm{s}$, respectively.

\section{Results and discussions}

\subsection{Microstructure tests}

The SEM images of the surfaces of the wing membrane of the dragonfly reveal that along the direction of the thickness the membrane is divided into three layers, which are separately referred to as dorsal surface, middle layer and ventral surface. The thickness of them was measured by the IAS and statistically given to be $513.63 \pm 69.02 \mathrm{~nm}, 1.93 \pm 0.18 \mu \mathrm{m}$ and $356.33 \pm 42.50 \mathrm{~nm}$ (mean \pm standard deviation), respectively. And the average gross thickness of the wing membrane was simultaneously measured to be about $2.8 \pm 0.3 \mu \mathrm{m}$, which was in good agreement with the traditional results [8,9], as shown in Fig. 2A. In addition, both the dorsal and the ventral surfaces, as well as the surface of the wing venations, were found to be composed of numerous nanometer scale columns coated by the cuticle wax secreted. These columns almost vertically stood up and randomly distributed on the surfaces of the membranes, and the diameter and the numerical density of the columns on the surfaces were measured to be about $38.52 \pm 5.88 \mathrm{~nm}$ and $75 \pm 7 \mu \mathrm{m}^{-2}$, respectively, as shown in Fig. 2B. The heights of the columns on the dorsal and the ventral layers are equal to the thickness of the two layers, respectively. From the numerical density, we readily estimated the average distance between two columns on the surface to be about $115 \mathrm{~nm}$. Further, it was observed that the top of each of the nanometer scale columns revealed the shape of a hemispherical cap, the diameter of which was approximately equal to that of the columns. In particular, it was found that there were some nanometer scale grooves symmetrically engraved on the surface of each of the columns along the direction of the length of the columns, as shown in the inset of Fig. 2B, and the thickness of the grooves was measured coarsely $13.8 \pm 2.6 \mathrm{~nm}$.

To describe the surface characteristics of the membrane, we obtain the random radial distribution function of the columns on the surface of the membrane, $p(r)$, as

$p(r) \mathrm{d} r=n \mathrm{e}^{-\pi n r^{2}} 2 \pi r \mathrm{~d} r$ 
(A)

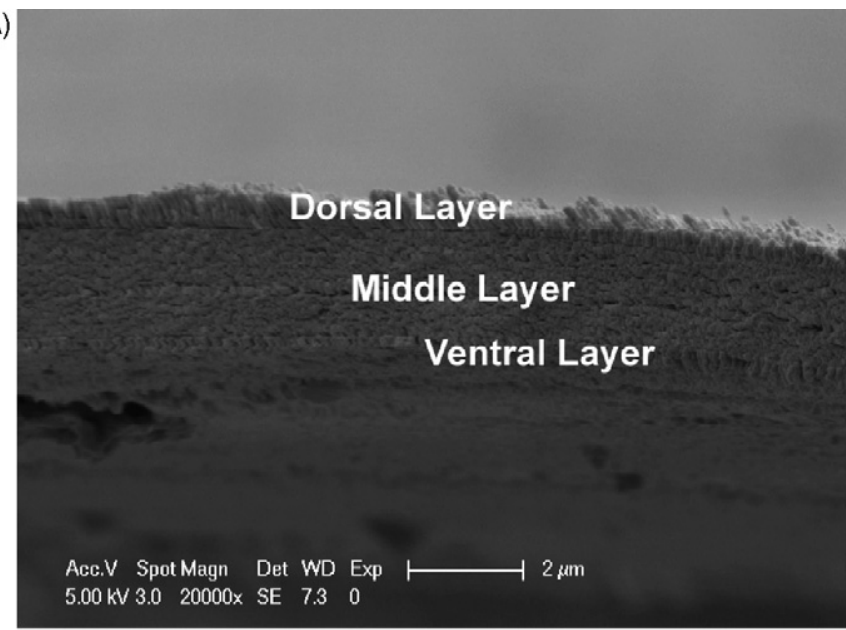

(B)

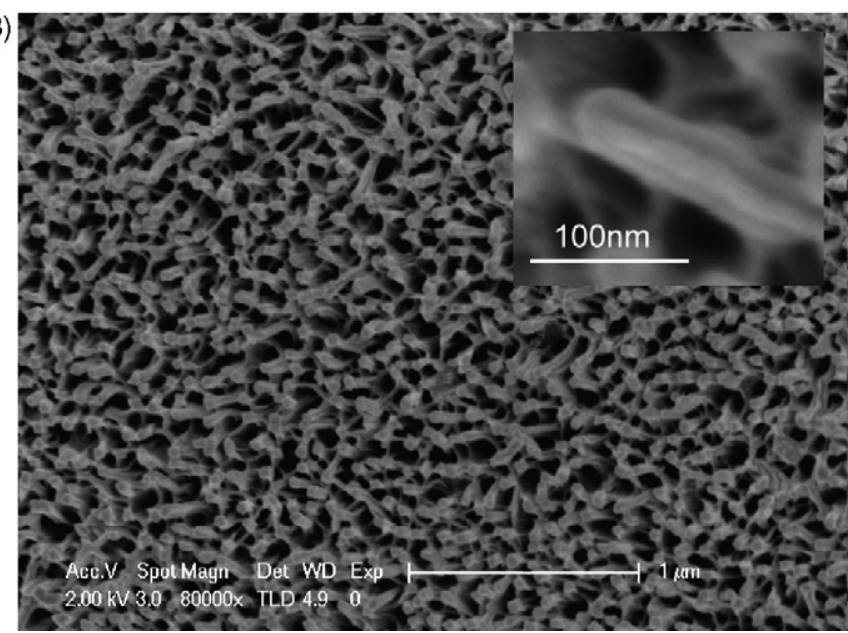

Fig. 2. (A) SEM image showing a cross-sectional surface of the wing membrane, which includes three layers, i.e. dorsal surface, middle layer and ventral surface, respectively. (B) SEM image showing the surface view of the cuticle waxy layer Inset of (B), the top of a column displays the shape of a spherical cap and some nanogrooves are symmetrically engraved on the surface of the column.

where $n=75 \pm 7 \mu \mathrm{m}^{-2}$ is the numerical density as given above, $p(r) \mathrm{d} r$ is the probability to find the closest column within $r$ and $r+\mathrm{d} r$. The radial, $r$, is the distance from the center of one column to that of the closest column. Therefore, the average distance between two columns is written as

$\langle r\rangle=\int_{0}^{\infty} r p(r) \mathrm{d} r=\frac{1}{2 \sqrt{n}}$

According to Eq. (8), we obtain the average distance from the center of column to another between nearest-neighbor columns to be $\langle r\rangle=58.2 \pm 2.7 \mathrm{~nm}$, which is just half as large as the average distance between two columns on the surface.

In addition, because the surface microstructure of the membrane displays a random nanometer scale roughness $[17,18]$, we briefly study the hydrophobicity of the surface of the membranes. By using sessile water-drop measurements, we measured the contact angles of water droplets on the surface of the different cells and obtained the average contact angle of the membranes with water droplets to be $174.21 \pm 2.19^{\circ}$. In particular, the maximum water contact angle measured in the experiments reached

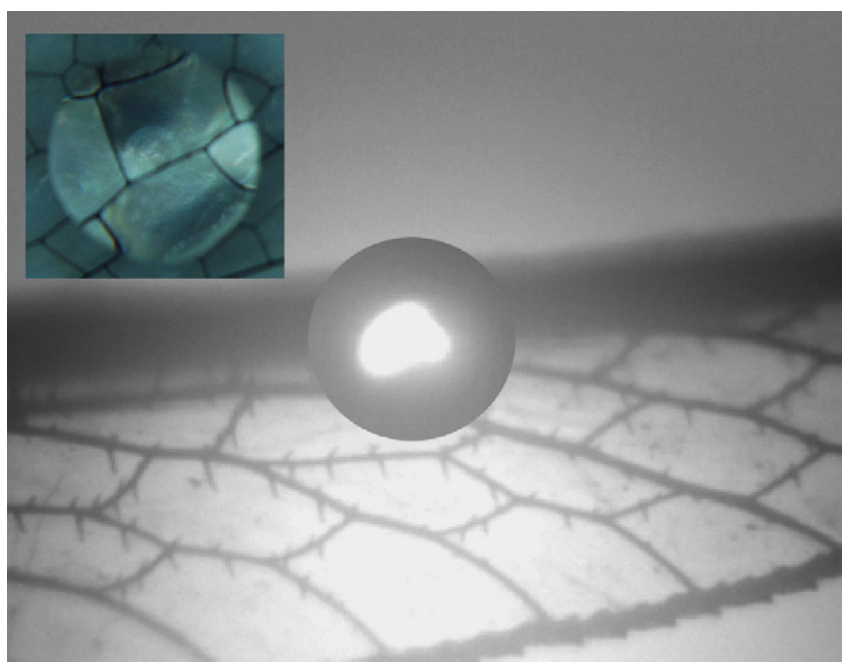

Fig. 3. Pictures showing an almost ball-shaped water droplet on the surface of the wing membrane of a dragonfly. Inset, the top view of a water droplet on the wing membrane shows a small contact region between water droplet and the surface.

$177.2^{\circ}$, which very closely approached the theoretical limit of a water contact angle, $180^{\circ}$, as shown in Fig. 3. Such the superhydrophobicity makes the dragonfly fly agilely in the rain and keep its wings non-wetting and self-cleaning, furthermore, reduce drag during flight.

According to Cassie and Baxter equation for wetting on the surface with a heterogeneous structure composed of solid and air, the apparent contact angle $\theta^{*}$ of a water droplet on the membrane surface of the dragonfly wings can be written by [17]:

$\cos \theta *=-1+\phi_{\mathrm{s}}(\cos \theta+1)$

where $\phi_{\mathrm{s}}$ is the fraction of projected plane area of water in contact with the membrane surface of the wing and $\theta$ is the equilibrium contact angle between water droplet and the surface of the cuticle wax covered on the membrane surface of the wing and is approximately equal to $105^{\circ}$ [17]. When a water droplet contacts with the surface of the dorsal layer of the wing membrane, its contact region with each of the nanometer scale columns in the dorsal layer should not be whole of the spherical cap of its top because the cuticle wax itself is hydrophobic and the diameter of the spherical cap is too small. However, this contact area and shape are not directly measured by the experiments. Here we coarsely estimate the value of the contact area. It is assumed that the shape of the contact area approximates a small spherical cap covered on the spherical top of the column and its diameter is taken as the value that the diameter of the column subtracts the thickness of the two grooves opposite to one another. So that, we can compute the area of the contact region between a water droplet and a column to be about $129.5 \mathrm{~nm}^{2}$ and the fraction of the contact area of a water droplet with the surface of the dorsal layer, $\phi_{\mathrm{s}}$, to be about $0.546 \%$. According to the equation above, the apparent contact angle is computed to be approximately $174.8^{\circ}$, which is in good agreement with the average experimental value obtained. This is an indication that available air is trapped in spaces in the microstructure of the surfaces of the dorsal and ventral layers, and the grooves on the 


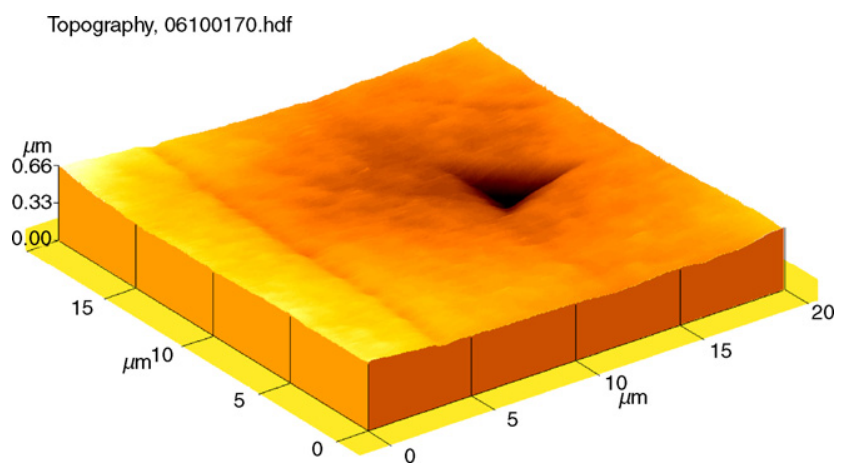

Fig. 4. Atomic force microscope image of nanoindentation showing the indented shape and thickness made by a triangular pyramid Berkovich indenter.

columns efficiently increase the hydrophobicity of the dragonfly wings.

\subsection{Mechanical tests}

Based on the microstructure given above, on the one hand, there is a column layer of about $513 \mathrm{~nm}$ thickness on the dor-

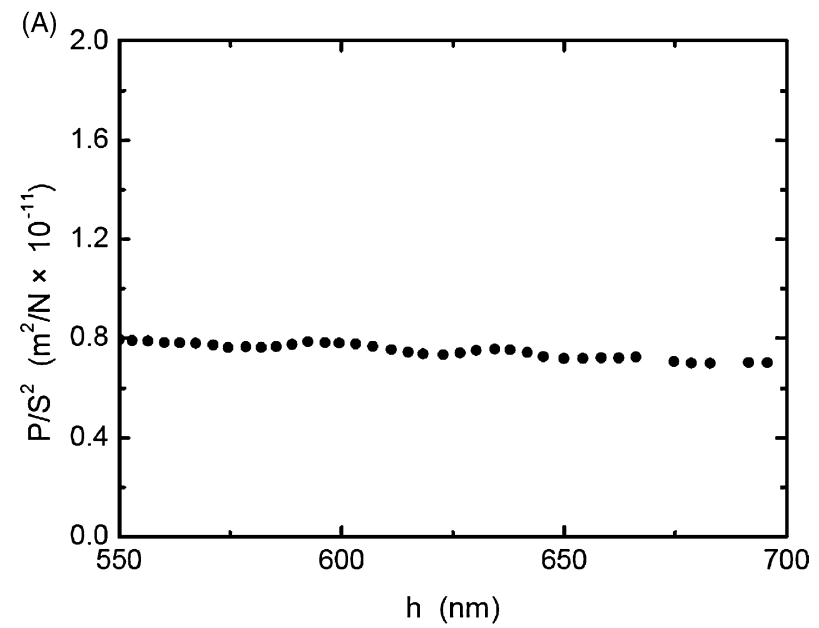

(B)

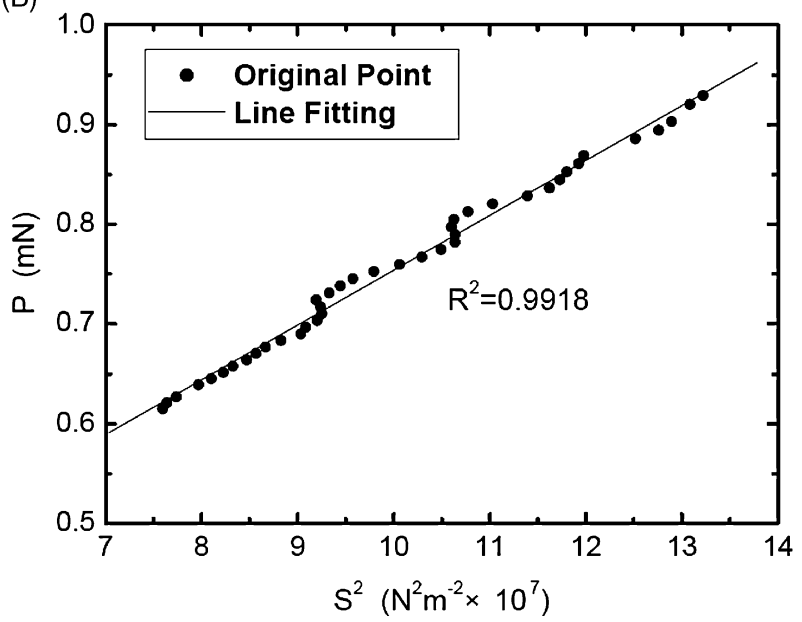

sal surface of the wing membrane. So, when we employed the indenter to measure the material properties of the membrane, the penetration depth of the tip of the indenter should be greater than the thickness of the column layer. On the other hand, the middle layer and the ventral surface of the wing membrane are about 1.93 and $0.36 \mu \mathrm{m}$ thickness, respectively. To ensure that the effects of the substrate are insignificant, the indentation depth should be not greater than $10 \%$ of the tested sample thickness, i.e. $229 \mathrm{~nm}[10,13]$. Therefore, we accurately controlled the indentation depth and only considered the testing data that the indentation depth varied from 550 to $700 \mathrm{~nm}$ as the effective data in the analysis, as shown in Fig. 4.

According to Eqs. (1) and (6), we readily obtain a relationship between the indentation load and the contact stiffness as

$$
\frac{P}{S^{2}}=\frac{\pi H}{4 \beta^{2} E_{\mathrm{r}}^{2}}
$$

In Eq. (10), the right hand side of the equation is only composed of material constants. This is an indication that the left hand side of the equation, $P / S^{2}$, is thoroughly determined by the material constants, which should not change with respect to the penetra-
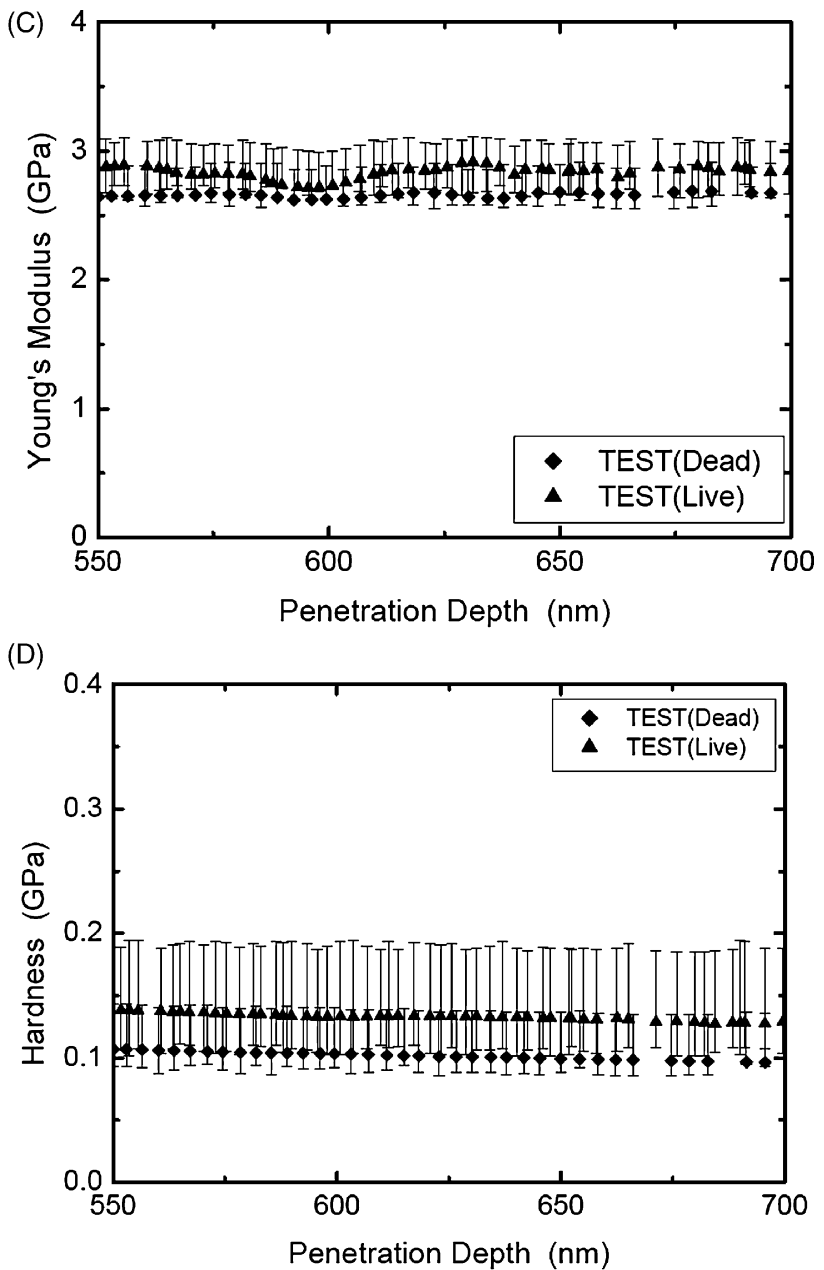

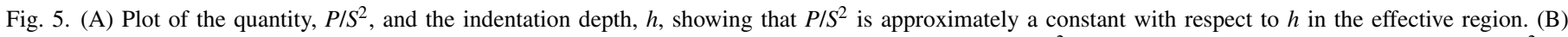

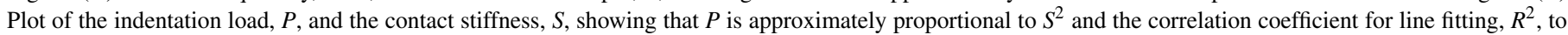

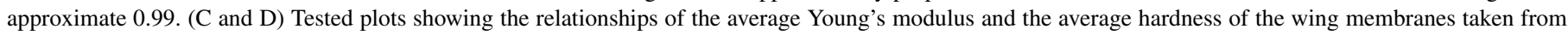
the living and dead dragonflies with respect to the change of the indentation depth in the effective region, respectively. 
tion depth $h$. Therefore, Eq. (10) can be employed to check the reliability of the test data and to control the testing systematic errors $[13,14]$.

Fig. 5A shows that $P / S^{2}$ is approximately a constant with respect to the indentation depth $h$ in the effective region from 550 to $700 \mathrm{~nm}$, and Fig. 5B shows that $P$ is approximately proportional to $S^{2}$ with the correlation coefficient for line fitting, $R^{2}$, to approximately reach 0.99 . According to Eq. (10), these indicate that the testing data obtained under these conditions are valid for the tested materials. Fig. 5C and D separately display the relationships of the average tested Young's modulus and the average tested hardness of the wing membranes taken from living and dead dragonflies with respect to the indentation depth from 550 to $700 \mathrm{~nm}$. The experimental results give that the Young's moduli of the membranes of living and dead dragonflies are $2.85 \pm 0.23$ and $2.74 \pm 0.28 \mathrm{GPa}$, respectively, and the hardness of the wing membranes of the living and the dead are $0.14 \pm 0.04$ and $0.10 \pm 0.03 \mathrm{GPa}$, respectively.

The results stated above on the Young's modulus of the wing membranes of both living and dead dragonflies are greater than the previous result, $1.5 \mathrm{GPa}$, obtained by the same test method, namely, the nanoindentation technique [9]. It may be because the effects of the column layer of the wing surface were not considered in the previous measurements. In order to prove the present results, we investigated the Young's modulus of the membranes under the condition that the effects of the surface columnar layer were not considered in our experiments. We tested 20 membrane samples involving separately 10 samples of the living and the dead under the condition. The Young's modulus of the living and the dead samples were measured to be $1.41 \pm 0.44$ and $1.37 \pm 0.75 \mathrm{GPa}$, respectively, which were in approximate agreement with the previous results obtained by the same method [9]. It was reasonably believed that the discrepancy in value between the present and the previous results comes from controlling the depth of the nanoindentation rather than differences in technique, equipment, etc.

In addition, it was found that the Young's modulus of the wing membrane of the dragonfly was lower than that of locust (5 GPa), $\alpha$-keratin (4 Gpa), $\beta$-keratin (8-10 Gpa), lepidopteran silk (10 Gpa) [4], and cicada (3.7 GPa) [12], but was much higher than some amorphous protein polymers, e.g. resilin (1.2 MPa) and abductin (4 MPa) [19], where the Young's modulus of locust was measured by the traditional method of tensile testing and the Young's modulus of cicada was measured by both the tensile testing and the nanoindentation methods $[4,12]$. In particular, the present results indicate that there are only a few differences between the wing membranes taken from living and dead dragonflies. From the results above, the relative errors of both the mean Young's modulus and the mean hardness for the living and the dead wings are computed to be less than $4 \%$. However, under the tested conditions that we measured the mechanical properties of the membranes, the testing processes might induce a testing error to be about $5 \%[15,16]$. Therefore, if we consider the testing deviations derived from the testing system itself, such as the instability of the indenter, we can approximately deem that there are not differences between the material properties of the wing membranes of the living and the dead dragonflies. How- ever, it is a fact that the wings of dead insects are much more brittle than those of the living. This is an indication that the differences between the mechanical properties of the wing of living and dead insects do not derive from their wing membranes.

\section{Conclusions}

The present work studies the microstructure and material properties of the wing membrane of the dragonfly. In the direction of the thickness, the membrane is divided into three layers that are separately referred to as dorsal surface, middle layer and ventral surface, the average thickness of which are measured to be $513.63 \mathrm{~nm}, 1.93 \mu \mathrm{m}$ and $356.33 \mathrm{~nm}$, respectively. On the dorsal and ventral surfaces, the membrane displays a random distribution rough microstructure that is composed of numerous nanometer scale columns coated by the cuticle wax. The average diameter and numerical density of the columns on the surfaces are about $38.52 \mathrm{~nm}$ and $75 \mu \mathrm{m}^{-2}$, respectively. According to the characteristics of the surfaces, we present a statistical radial distribution function of the columns on the surfaces, $p(r)=2 \pi r n \mathrm{e}^{-\pi n r^{2}}$, and give the average distance between nearest-neighbor columns to be about $58.2 \mathrm{~nm}$ from the distribution function. Also, the microstructure of the surface of the membrane has excellent superhydrophobicity with the water contact angle about $174^{\circ}$. Based on the surface microstructure of the membrane, the average Young's modulus and hardness of the membrane are measured by the nanoindentation technique to be about 2.85 and $0.14 \mathrm{GPa}$, respectively. When we consider the deviations derived from the testing system, we indicate that there are few differences of the material properties between the membranes taken from the wings of living and dead dragonflies. The present results can provide a guide to the biomimetic designs of the aerofoil materials of microair vehicles.

\section{Acknowledgments}

The authors are very grateful to the reviewers for their suggestions and comments. We thank Dr. T.H. Zhang, and J.J. Xie, for their help on the experiments. This work was supported by the National Natural Science Foundations of China (grant 10672164 and 10372102) and CAS Innovation Program (grant KJCX2-YW-M04).

\section{References}

[1] S.A. Combes, T.L. Daniel, J. Exp. Biol. 206 (2003) 2979-2987.

[2] S.A. Combes, T.L. Daniel, J. Exp. Biol. 206 (2003) 2989-2997.

[3] R.J. Wootton, Annu. Rev. Entomol. 37 (1992) 113-140.

[4] C.W. Smith, R. Herbert, R.J. Wootton, K.E. Evans, J. Exp. Biol. 203 (2000) 2933-2943.

[5] R.J. Wootton, Sci. Am. (1990) 114-120.

[6] S. Ho, H. Nassaf, N. Pornsin-Sirirak, Y.-C. Tai, C.-M. Ho, in: I. Grant (Ed.), Proceedings of the International Council of the Aeronautical Sciences (ICAS), ICAS, Toronto Canada, 2000, pp. 551.1-551.10.

[7] R.S. Fearing, K.H. Chiang, M.H. Dickinson, D.L. Pick, M. Sitti, J. Yan, in: D.L. Alessandro (Ed.), Proceedings of the 2000, IEEE. International Conference on Robotics and Automation, San Francisco, CA, Wiley \& Sons, New Jersey, 2000, pp. 1509-1516.

[8] D.J.S. Newman, R.J. Wootton, J. Exp. Biol. 125 (1986) 361-372. 
[9] P. Kreuz, B. Kesel, W. Arnold, H. Vehoff, W. Nachtigall, in: W. Nachtigall, A. Wisser (Eds.), Biona-Report 14, Fischer, Stuttgart, 2000, pp. 1617.

[10] W.C. Oliver, G.M. Pharr, J. Mater. Res. 7 (1992) 1564-1583

[11] S.E. Morgen, R. Misra, P. Jones, Polymer 47 (2006) 2865-2873.

[12] F. Song, K.L. Lee, A.K. Soh, F. Zhu, Y.L. Bai, J. Exp. Biol. 207 (2004) 3035-3042.

[13] D.L. Joslin, W.C. Oliver, J. Mater. Res. 5 (1990) 123-126.
[14] R. Saha, W.D. Nix, Acta Mater. 50 (2002) 23-38.

[15] T.H. Zhang, Y. Huan, Tribol. Lett. 17 (2004) 911-916.

[16] T.H. Zhang, Y. Huan, Compos. Sci. Technol. 65 (2005) 1409-1413.

[17] A. Lafuma, D. Quéré, Nat. Mater. 2 (2003) 457-460.

[18] R. Blossey, Nat. Mater. 2 (2003) 301-306.

[19] J.M. Gosline, in: J.F.V. Vincent, J.D. Currey (Eds.), The Mechanical Properties of Biological Materials, Cambridge University Press, Cambridge, 1988, pp. 331-358. 